\title{
A real-time passive vision system for robotic arc welding
}

Liu, Jinchao; Fan, Zhun; Olsen, Søren Ingvor; Christensen, Kim Hardam; Kristensen, Jens Klæstrup

Published in:

2015 IEEE International Conference on Automation Science and Engineering (CASE)

DOI:

10.1109/CoASE.2015.7294109

Publication date:

2015

Document version

Peer reviewed version

Citation for published version (APA):

Liu, J., Fan, Z., Olsen, S. I., Christensen, K. H., \& Kristensen, J. K. (2015). A real-time passive vision system for robotic arc welding. In 2015 IEEE International Conference on Automation Science and Engineering (CASE) (pp. 389-394). IEEE. https://doi.org/10.1109/CoASE.2015.7294109 


\title{
A Real-time Passive Vision System for Robotic Arc Welding
}

\author{
Jinchao Liu, Zhun Fan, Senior Member, IEEE, Søren Ingvor Olsen, \\ Kim Hardam Christensen, Jens Klæstrup Kristensen
}

\begin{abstract}
Visual sensing in arc welding has become more and more important. However, it has remained a challenging problem due to the harsh environment, especially the interference of the extremely strong arc light. A typical industrial solution for seam detection is to use laser scanner which however still suffers from several limitations. Solutions for weld pool sensing mostly rely on high speed cameras and powerful external illuminations and therefore the wide use in industrial environments is limited. In this paper we present a real-time passive machine vision system for both seam tracking and weld pool sensing in robotic arc welding. The system, which consists of a CCD camera and a carefully-selected narrowband pass filter, is capable of extracting the geometrical profile of the seam that is close to the arc region and the weld pool boundary simultaneously in real time. This visual information can be further utilized by the control system to navigate the welding robot and adjust the welding parameters. Experiments including several real-time demonstrations have been made and verified the feasibility of the proposed vision system.
\end{abstract}

Index Terms - Vision system, visual sensing, active contours, arc welding automation, weld pool sensing.

\section{INTRODUCTION}

For decades the need of robotic arc welding has become more and more intensive. Many researchers and engineers have devoted their effort to automating the arc welding process in order to increase the productivity and quality, decrease the exposure of the human welders to the harmful environments. However, it still remains a challenging problem to obtain a closed-loop control of the welding process. The reasons are twofold. One is the welding process is rather complex and difficult to be modeled. Design of the control strategy is therefore challenging. The other is there is still a lack of proper sensor systems which can extract sufficient information of the welding process. More specifically, the system should be able to detect the joint location and geometrical profile and the weld pool information such as shapes. The former is commonly referred to as "seam detection" and the latter "weld pool sensing". For years, different kinds of sensors for seam detection and weld pool sensing were

J. Liu was with the Technical University of Denmark and FORCE Technology, Denmark. He is now with VisionMetric Ltd, Canterbury, Kent, UK. liujinchao2000@gmail.com

Z. Fan is with Guangdong Provincial Key Laboratory of Digital Signal and Image Processing and Department of Electronic Engineering, Shantou University. 515063, Shantou, China. zfanestu.edu.cn

S. Olsen is with Department of Computer Science, University Of Copenhagen, 2100 Copenhagen, Denmark. ingvorediku.dk

K. Christensen is with Division of Welding \& Production Innovation, FORCE Technology, DK-2605 Brondby, Denmark. kmc@ force.dk

J. Kristensen was with Division of Welding \& Production Innovation, FORCE Technology, DK-2605 Brondby, Denmark. jens@klaestrup.dk developed, such as the contact probe, temperature sensors, through-the-arc sensors, electromagnetic sensors, optical sensors and sound sensors and so on.

Among all these sensors, optical sensors possess the advantage of receiving the visual information and draw more and more attention in recent years. For example, a typical industrial sensor solution for seam tracking is to use laser scanner. It permits us to have the accurate measurement of the joint location and geometry[1]. Although laser scanner has been widely used for seam tracking, it still suffers from several limitations. First, the laser scanner is still relatively expensive. Second, it must be placed at a distance to the molten pool. Third, it may cause problems when dealing with shiny surfaces. It should be pointed out that laser scanner solution is typically not able to sense the weld pool.

In [2], Shi et al. proposed an efficient weld seam detection algorithm which is able to handle most shapes of weld seams in butt joint welding. The system can only work before welding. In [3], Shen et al. developed a welding robot system in square-wave alternating current gas tungsten arc welding based on the real-time visual measurement. The system measures the offset of the torch to the seam center and the width of the seam gap and navigate the torch movement. In [4], [5], Zhang et al. proposed a vision-based sensing system for weld pool monitoring. A dot-matrix pattern of laser light was projected onto the weld pool surface and the reflected light was captured by an imaging plane. By analyzing the distortion of the reflected dot-matrix, the surface of the weld pool could be recovered. However, the proposed methods are off-line and can not work in real time. In [6], Bae et al. developed a visual sensing system for automatic gas metal arc welding of the root pass of a steel pipe. The system is equipped with a CCD camera and optical lens to capture weld pool images. By triggering the camera in the shortcircuit moment, the proposed system is able to obtain weld pool images without any interference of the arc light. This simplifies the task of extracting weld pool boundary. The limitation is that the vision system can only work in shortcircuit mode of arc welding.

In this paper, a camera-based sensor system without using external illuminations for seam detection and the weld pool sensing is presented. The system consists of a CCD camera and a narrow bandpass filter selected by maximizing the signal-to-noise ratio through spectrum analysis. The exposure time of the camera is also altered on the fly in order to obtain images for different purposes, i.e., seam detection or weld pool sensing. Two image analysis algorithms were also proposed to extract corresponding information. More 
specially, a seam detection algorithm based on RANSAC and Kalman filter was proposed to extract the four lines that describe the seam in the case of v-groove. For the weld pool boundary tracking, we proposed an active contour model driven by a Bayesian classifier to track and model the weld pool boundary. These geometrical information can then be used to realize an adaptive closed-loop control system. Experiments including several realtime demonstrations were made and proved the feasibility of the proposed system.

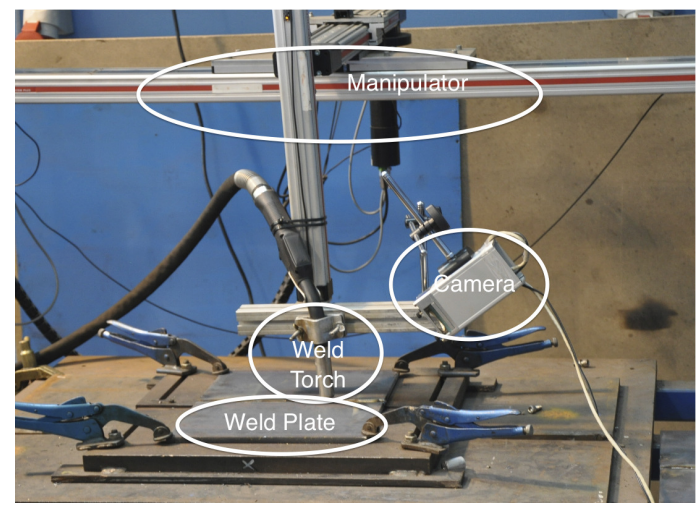

Fig. 1. The configuration of the experiment platform, including an industrial camera equipped with optical filters, a manipulator and a welding torch.

The rest of the paper is organized as follows. In section 2 , the overall architecture of the proposed vision system is presented. In section 3, the image analysis algorithms are discussed in detail. Section 4 presents the experiments of applying the proposed vision system and discusses the results. In the end we will conclude the paper and discuss the future work.

\section{The ARChitecture OF THE Proposed Vision SYSTEM}

From the hardware side, the proposed vision system consists of a monochrome CCD camera and a carefully-selected narrow bandpass filter. Fig. 1 shows the experimental setup. The software system has two major modules, the camera control module and the image analysis module, as shown in Fig. 2. Detailed discussion is presented as follows.

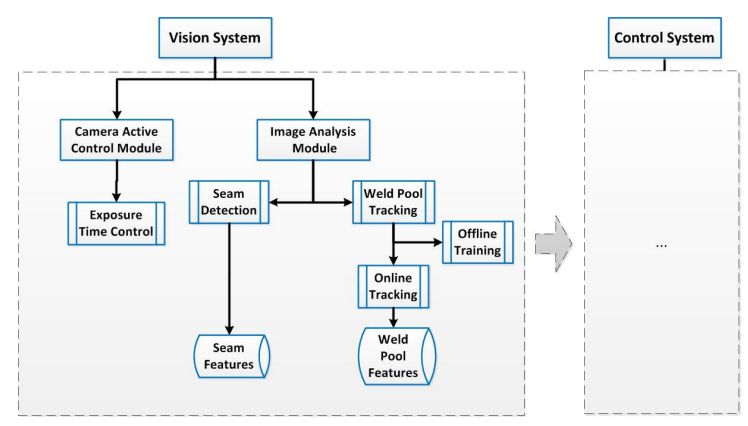

Fig. 2. The architecture of the proposed vision system.

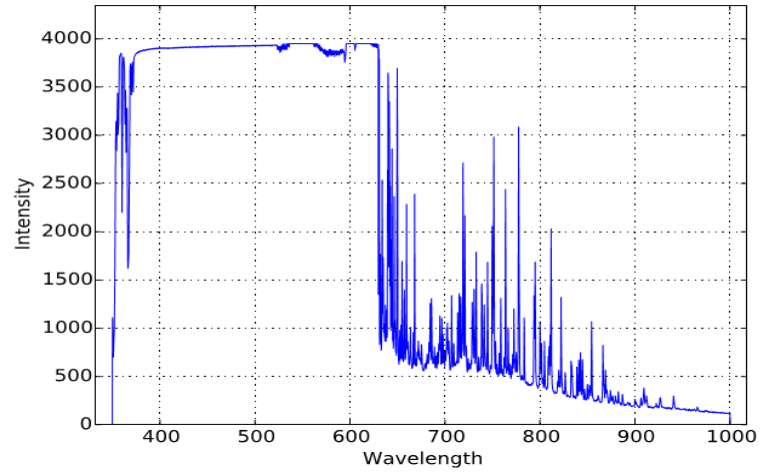

(a) Arc light spectrum

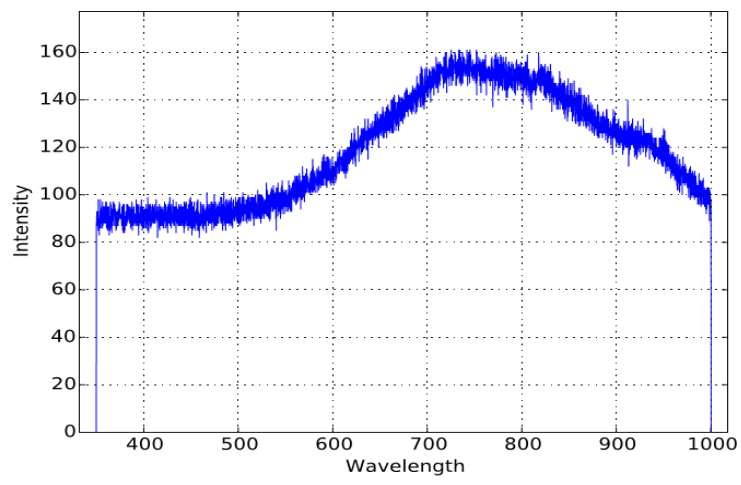

(b) Weld pool spectrum

Fig. 3. The spectrums of the light generated during welding. $x$ axis represents the wavelength, y axis represents the magnitude. (a) Typical spectrum of the arc light. In this case, the magnitude of the weld pool light is negligibly small compare to the arc light. (b) Typical spectrum of the weld pool light.

\section{A. The Selection of Optical Filters}

The biggest challenge that one has to face for visual sensing in arc welding is how to deal with the interference of the extremely-high intensity arc light. In this paper this problem is tackled by means of optical filters and advanced image analysis techniques.

Fig. 3 shows the spectrum of the lights in a weld. It was captured by a spectrometer during welding using an exposure time of $100 \mathrm{~ms}$. The distance from the optical probe of the spectrometer to the weld pool is approximately $10 \mathrm{~mm}$. It shows that the region in the spectrum that is mainly composed of the weld pool light is separate from the region of the arc light, in spite of that the intensity of the arc light is very high. This motivates and permits us to use a narrow bandpass filter to decrease the intensity of the arc light and meanwhile increase the intensity of the weld pool light passing into the camera. In order to increase the signal-to-noise ratio, the center of the bandpass filter should locate within the interval of around $700 \mathrm{~nm}$ to $1000 \mathrm{~nm}$. In this proposed system, a narrow bandpass filter with a center wavelength of $780 \pm 10 \mathrm{~nm}$ was employed. In addition, a standard protection glass was also used to protect the 


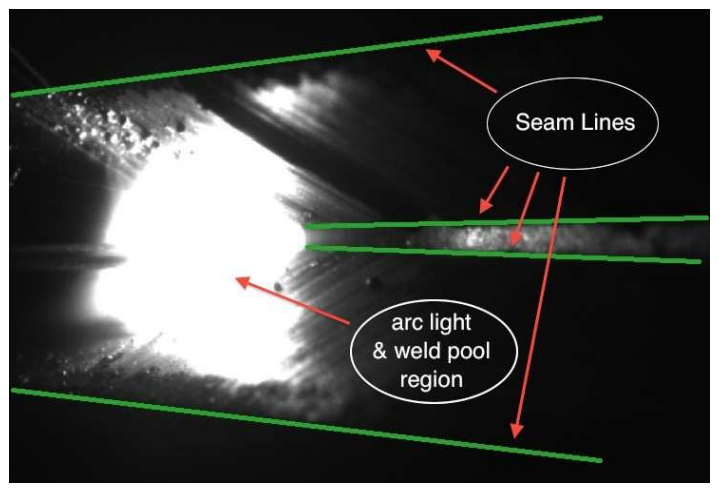

(a)

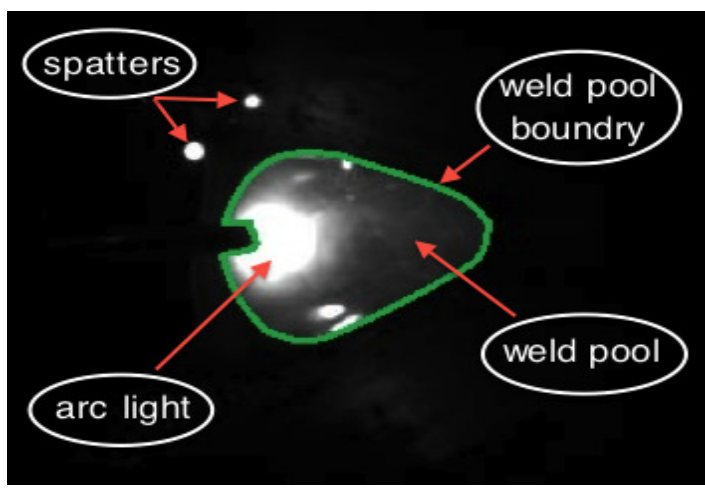

(b)

Fig. 4. (a) A typical frame captured for seam detection. Four lines that describe the V-Groove workpiece are marked in green. (b)A typical frame for weld pool tracking. The weld pool boundary is manually annotated here and marked in green.

bandpass filter from the spatters produced during welding.

\section{B. The Active Control of the Camera}

The camera control module is responsible for controlling the exposure time of the camera on the fly. The reason is that seam detection and the weld pool sensing require different illumination conditions. In the case of seam tracking, we need some but not too much arc light to illuminate the seam. On the other hand, weld pool sensing requires that the arc light should be reduced as much as possible. Changing the exposure time on the fly, long exposure time for seam detection and short exposure time for weld pool sensing, can meet these two conflicted requirements.

\section{Image Analysis Algorithms for SeAm Detection AND Weld PoOl TRACKING}

With the hardware system as well as the exposure time control strategy, proper images can be obtained for the purpose of seam detection or weld pool tracking. Two typical frames are shown in Fig. 4. The desired geometrical features of seam detection and weld pool tracking are manually annotated in green. In this section, two image analysis algorithms for extracting these desired geometrical features are presented.

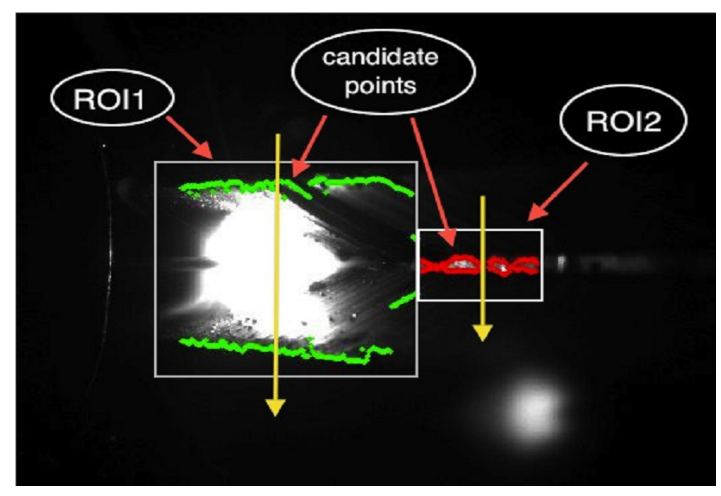

Fig. 5. An illustration of the seam detection algorithm. Two image ROIs are calculated by computing the image moments. Candidate points are then extracted within each ROI by searching the first and the last edge points along the vertical direction.

\section{A. The Seam Detection Algorithm}

As shown in Fig. 4(a), four lines that describe the shape of V-Groove workpiece are the desired features that should be extracted from the images in real time. Therefore, we propose a line detection algorithm based on RANSAC and Kalman filters for seam detection.

The typical procedure of line detection is to extract the candidate points first and then fit lines based on these points. In our application, illuminating the seam only relies on the arc light since there is no auxiliary light sources. Due to the dramatically change of the illumination, the candidate points are not very well defined by local structure and therefore contain many outliers. RANSAC[7] is a natural choice for robust line fitting which can tolerate a large portion of outliers.

The pseudo-code of the proposed seam detection algorithm can be found in algorithm 1. First, the image ROIs, i.e., two rectangles are obtained by computing the image moments. As shown in Fig. 5, ROI 1 covers the overall weld pool area and serves the purpose of extracting two outer lines of the seam. $R O I_{2}$ is placed just ahead the weld pool area for extracting the two inner lines of the seam. Second, edge points for line fitting are computed via canny edge detector. As discussed earlier, the candidate points here are not well defined by for example local gradient but global structure due to the dramatic change of illumination during welding. Therefore, we define the candidate points as the first or last edge points in the scan line which is along the vertical direction according to our camera setup. This column search is performed for each column of each ROI and produce a series of candidate points as shown in Fig. 5. A robust line fitting based on RANSAC are then carried out based on these candidate points.

\section{B. The Weld Pool Boundary Tracking Algorithm}

In arc welding, the shape of the weld pool and its position relative to the seam play an important role in navigating the torch and adjusting welding parameters in order to obtain a good quality welding. Therefore, the ability of tracking the weld pool boundary in real time becomes crucial. A typical 


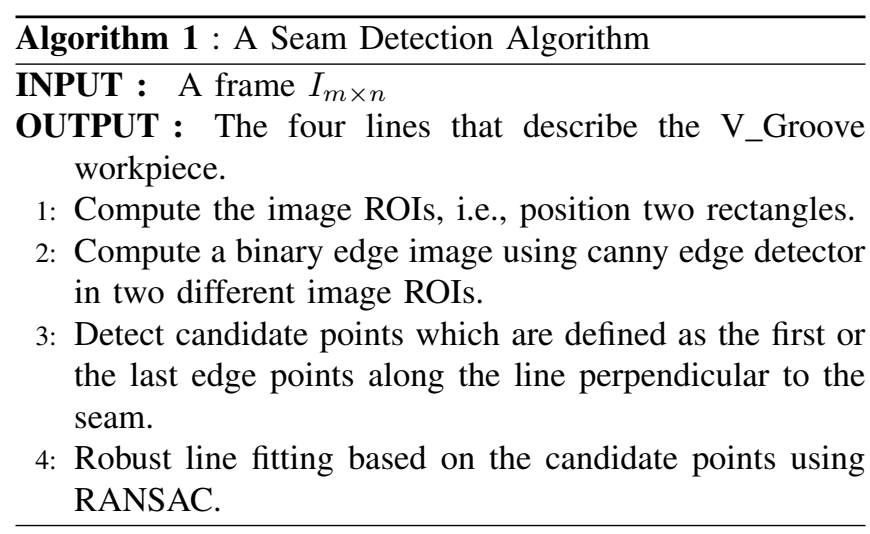

frame captured by the camera system is displayed in Fig. 4(b) where the weld pool boundary is manually annotated. It was found in this application that low-level approaches such as thresholding or edge detection do not yield satisfactory performance. This is mainly due to the interference of the varying arc light. Very often the detected edge points do not form a closed contour or even worse, are completely wrong. In order to obtain a satisfactory result, global information or prior knowledge ought to be taken into account. Active contours, or snakes[8] offer an elegant framework where prior knowledge can be added. Therefore, in this paper we propose a visual tracking algorithm for weld pool boundary tracking based on active contours.

In [9], [10] and [11], the concepts of region-based forces arising from the statistical modelling of different regions have been suggested. In [12], the authors proposed an active contour driven explicitly by supervised binary classifiers, more specifically a k-nearest neighbourhood, a support vector machine and a neural network. For each pixel, Haralick features were computed and used as the input of classifiers. Inspired by these works, we proposed to use a fast active contour driven by Bayesian classifiers for weld pool boundary extraction. Assume that the boundary $\mathcal{C}$ divides the whole image into two regions $\Omega_{i n}$ and $\Omega_{\text {out }}$. The class-conditional probabilities $p\left(I(\mathbf{x}) \mid \Omega_{\text {in }}\right)$ and $p\left(I(\mathbf{x}) \mid \Omega_{\text {out }}\right)$ are modeled using Gaussian mixture models. The prior probabilities $p\left(\Omega_{i n}\right)$ and $p\left(\Omega_{\text {out }}\right)$ are calculated according to the area ratio of the two regions. Thus the posterior probabilities can then be computed according to Bayes' theorem:

$$
p(\Omega \mid I(\mathbf{x})) \sim p(I(\mathbf{x}) \mid \Omega) * p(\Omega)
$$

where $\Omega \in\left\{\Omega_{\text {in }}, \Omega_{\text {out }}\right\} . I(\mathbf{x})$ denotes the intensity of the pixel $\mathbf{x}$. A Bayesian classifier $\mathcal{B}(\mathbf{x})$ can be formed and drives the control points of the active contour towards or away from the boundary. The evolving equation of the active contour using parametric representation is then given as follows:

$$
\frac{\partial \mathbf{x}}{\partial t}=-\lambda \cdot \mathcal{B}(\mathbf{x}) \cdot\left\|\frac{d \mathbf{x}}{d s}\right\| \hat{n}
$$

where $\lambda$ is a weighed factor. $\hat{n}$ is the normal unit vector pointing outward. $\mathcal{B}$ is a Bayesian classifier and defined as follows:

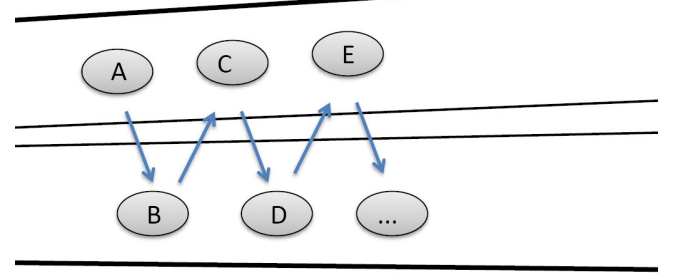

Fig. 6. An illustration of oscillating the torch with respect to the four seam lines. The torch follows the path $A \rightarrow B \rightarrow C \rightarrow \ldots$.

$\mathcal{B}(\mathbf{x})= \begin{cases}-1 & \text { if } \mathbf{x} \in \Omega_{\text {in }} \text { or } p\left(\Omega_{\text {in }} \mid I(\mathbf{x})\right)>p\left(\Omega_{\text {out }} \mid I(\mathbf{x})\right) \\ 1 & \text { if } \mathbf{x} \in \Omega_{\text {out }} \text { or } p\left(\Omega_{\text {in }} \mid I(\mathbf{x})\right)<p\left(\Omega_{\text {out }} \mid I(\mathbf{x})\right) \\ 0 & \text { if } \mathbf{x} \in \mathcal{C} \text { or } p\left(\Omega_{\text {in }} \mid I(\mathbf{x})\right)=p\left(\Omega_{\text {out }} \mid I(\mathbf{x})\right)\end{cases}$

\section{EXPERIMENTS AND DISCUSSION}

Experiments including several real time demonstrations have been made to evaluate the proposed vision system. The welding process employed in our application is MAG welding. V-Groove structure steel workpieces were used. The width of the seam is rather wide and not constant. Torch oscillation therefore became necessary in the experiments. The difficulty of seam detection and weld pools tracking is increased. The reason is twofold. One is the weld pool shape changes more dramatically during welding due to the torch oscillation. The other is in our setup the camera was mounted to the torch. As a consequence, the camera will then oscillate too. This gives rise to more drastic movement of the desired objects in the image domain.

Fig. 7 displays the results of seam detection. It can be seen that the four lines that describe the v-groove workpiece have successfully extracted. Two upper edges(two outer lines) and lower edges(two inner lines) of the v-groove are marked by red and green lines, respectively. For almost all frames, the extracted lines are rather accurate. It is worth noting that extracting the four lines are not the final goal, but provide the geometrical profile of the seam. For instance, for most cases, we are interested in the width of the upper edges or the lower edges, and the center line of the seam. A relatively high frame rate, in our application more than $10 \mathrm{~Hz}$, allows us to run a filtering algorithm such as Kalman filters afterwards to further stabilize these features.

Fig. 8 presents the results of the weld pool boundary tracking in a series of images. The initial contours marked in red circles were obtained by computing the image moments. The final contours marked in green captured the weld pool boundary successfully.

It is worth noting that the exact shape of the weld pool depends on the welding parameters as well as the workpiece and therefore do not have a single ground truth. The evaluation of the proposed vision system including both seam detection and weld pool tracking was done by welding experts inspection. 
The algorithm was implemented in $\mathrm{C}++$ and the speed of seam detection is around $10 \mathrm{~Hz}$, weldpool tracking $30 \mathrm{~Hz}$ on an ordinary PC.

\section{CONClusions AND Future WORK}

This paper presents a real-time passive vision system for both seam detection and weld pool sensing in arc welding. The system is capable of extracting the profile of the seam that is close to the high intensity arc region and the weld pool boundary in real time simultaneously. The system consists of a CCD camera and a carefully-selected narrow bandpass filter to capture frames for seam tracking and weld pool sensing respectively by changing the exposure time on the fly. To extract the geometrical profile of the seam, we proposed a seam detection algorithm based upon RANSAC. To track the shape of the weld pool, an active contour driven by a Bayesian classifier was proposed. Experiments of vgroove welding with torch oscillation were conducted and verified the feasibility of the proposed vision system. For future work, a control system will be developed based on these visual information to navigate the torch and adjust the welding parameters in real time to achieve automatic arc welding.

\section{REFERENCES}

[1] K. H. Christensen, "Arc welding automation process modelling \& control based on neural networks," Ph.D. dissertation, Technical University of Denmark, 2003.

[2] F. Shi, T. Lin, and S. B. Chen, "Efficient weld seam detection for robotic welding based on local image processing," Industrial Robot: An International Journal, vol. 36, pp. 277-283, 2009.

[3] H.-y. Shen, J. Wu, T. Lin, and S.-b. Chen, "Arc welding robot system with seam tracking and weld pool control based on passive vision," The International Journal of Advanced Manufacturing Technology, vol. 39, pp. 669-678.

[4] H. Song and Y. M. Zhang, "Measurement and analysis of threedimensional specular gas tungsten arc weld pool surface," Welding Journal, vol. 87, pp. 85.s-95.s, 2008.

[5] Z. Z. Wang, X. J. Ma, and Y. M. Zhang, "Simultaneous imaging and measurement of pool surface and metal transfer," Journal, vol. 90, pp. 121-128, June 2011.

[6] "An optical sensing system for seam tracking and weld pool control in gas metal arc welding of steel pipe," Journal of Materials Processing Technology, vol. 120, no. 1-3, pp. 458 - 465, 2002.

[7] M. A. Fischler and R. C. Bolles, "Random sample consensus: a paradigm for model fitting with applications to image analysis and automated cartography," Commun. ACM, vol. 24, pp. 381-395, June 1981.

[8] M. Kass, A. Witkin, and D. Terzopoulos, "Snakes: Active contour models," International Journal of Computer Vision, vol. 1, no. 4, pp. 321-331, 1988.

[9] T. Chan and L. Vese, "Active contours without edges," Image Processing, IEEE Transactions on, vol. 10, no. 2, pp. $266-277$, Feb. 2001.

[10] N. Paragios and R. Deriche, "Geodesic active regions: A new framework to deal with frame partition problems in computer vision," Journal of Visual Communication and Image Representation, vol. 13, no. 1-2, pp. $249-268,2002$.

[11] S. C. Zhu and A. Yuille, "Region competition: Unifying snakes, region growing, and bayes/mdl for multiband image segmentation," IEEE Trans. Pattern Anal. Mach. Intell., vol. 18, pp. 884-900, September 1996.

[12] J. Olivier, R. BonÃ̂l, J.-J. Rousselle, and H. Cardot, "Active contours driven by supervised binary classifiers for texture segmentation," in Advances in Visual Computing, ser. Lecture Notes in Computer Science, vol. 5358. Springer Berlin / Heidelberg, 2008, pp. 288-297. 


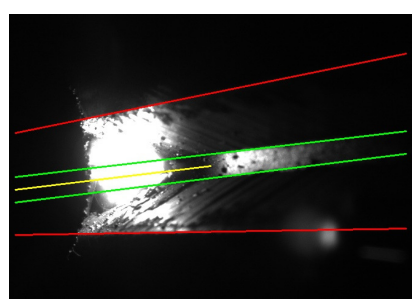

(a) Frame11

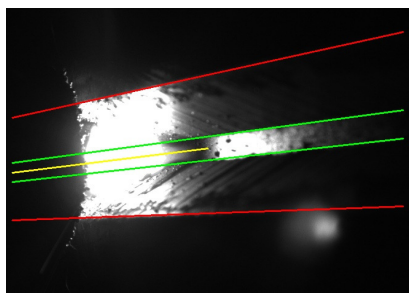

(e) Frame19

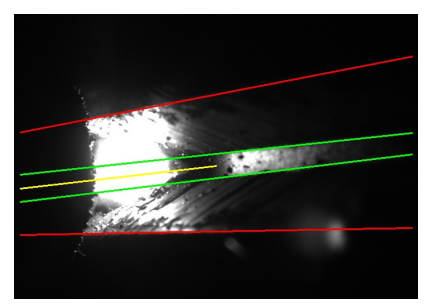

(b) Frame12

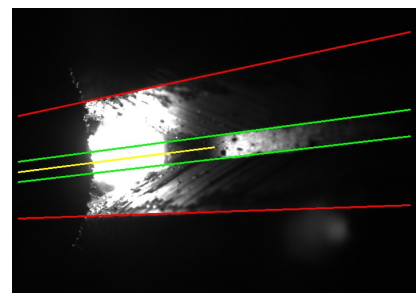

(f) Frame20

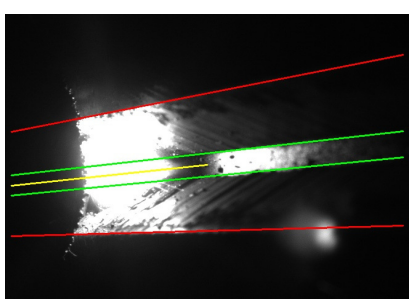

(c) Frame13

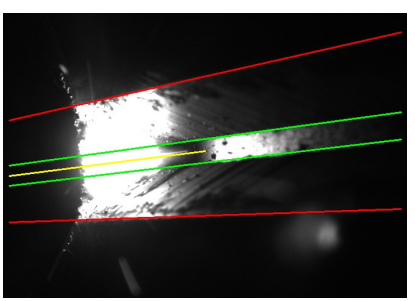

(g) Frame21

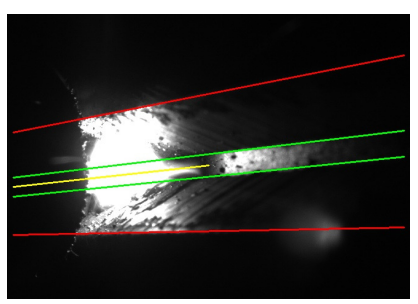

(d) Frame14

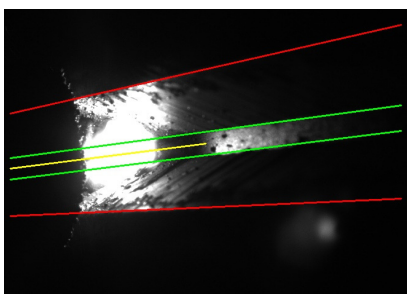

(h) Frame22

Fig. 7. Seam detection using the proposed method. The extracted four lines, two outer lines and two inner lines are marked in red and green respectively.

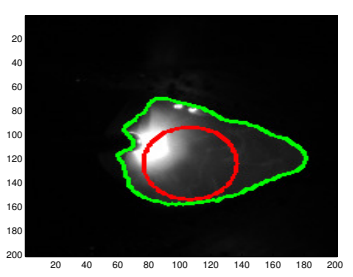

(a)

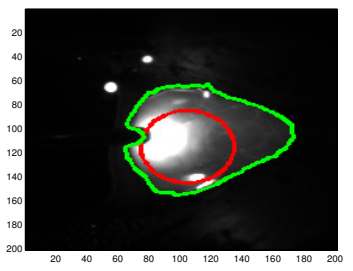

(e)

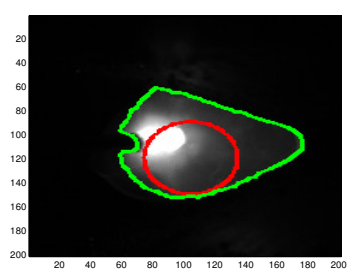

(b)

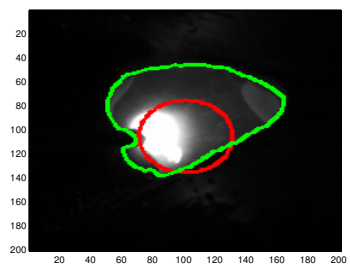

(f)

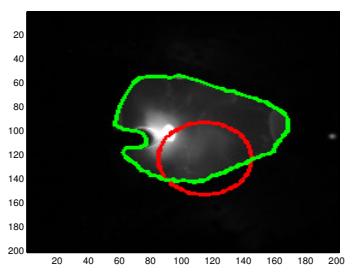

(c)

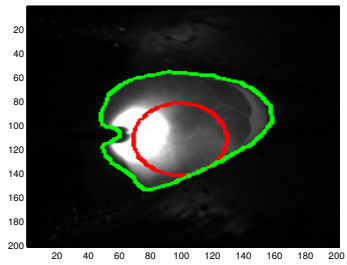

(g)

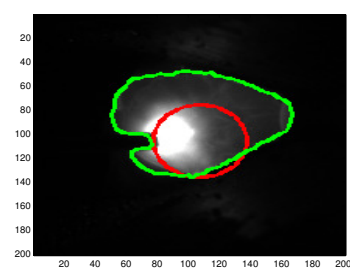

(d)

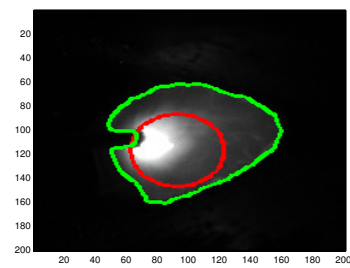

(h)

Fig. 8. Weld pool boundary extraction in a series of images captured during welding using the proposed method. Red circles indicate the initial positions of the active contours. The final curves are marked in green. Iterations 200. 